\title{
Non-target impacts of fungicide disturbance on phyllosphere yeasts in conventional and no-till management
}

\author{
Zachary A. Noel (iD ${ }^{1,4,5}$, Reid Longley (iD ${ }^{2,5}$, Gian Maria Niccolò Benucci (ID $^{1}$, Frances Trail ${ }^{1,3}$, Martin I. Chilvers ${ }^{1}$ and Gregory Bonito (D) ${ }^{1,2}{ }^{凶}$
}

(c) The Author(s) 2022

Fungicides reduce fungal pathogen populations and are essential to food security. Understanding the impacts of fungicides on crop microbiomes is vital to minimizing unintended consequences while maintaining their use for plant protection. However, fungicide disturbance of plant microbiomes has received limited attention, and has not been examined in different agricultural management systems. We used amplicon sequencing of fungi and prokaryotes in maize and soybean microbiomes before and after foliar fungicide application in leaves and roots from plots under long-term no-till and conventional tillage management. We examined fungicide disturbance and resilience, which revealed consistent non-target effects and greater resiliency under no-till management. Fungicides lowered pathogen abundance in maize and soybean and decreased the abundance of Tremellomycetes yeasts, especially Bulleribasidiaceae, including core microbiome members. Fungicide application reduced network complexity in the soybean phyllosphere, which revealed altered co-occurrence patterns between yeast species of Bulleribasidiaceae, and Sphingomonas and Hymenobacter in fungicide treated plots. Results indicate that foliar fungicides lower pathogen and non-target fungal abundance and may impact prokaryotes indirectly. Treatment effects were confined to the phyllosphere and did not impact belowground microbial communities. Overall, these results demonstrate the resilience of no-till management to fungicide disturbance, a potential novel ecosystem service provided by no-till agriculture.

ISME Communications; https://doi.org/10.1038/s43705-022-00103-w

\section{INTRODUCTION}

Disturbances from chemical applications in agriculture reduce the abundance of pests and pathogens and are common in modern agricultural ecosystems [1-5]. However, applying disturbance concepts to microbial communities can be challenging to assess recovery and analyze the full impacts of crop management. A lack of data on the impacts crop management combined with fungicide disturbances on the plant microbiome hinders developing novel strategies to minimize diversity loss, understand unintended consequences of these applications, and improve crop microbiomes' resilience. Observing fluctuations in taxa abundance and secondary effects mediated through microbial interactions following fungicide application opens the possibility for novel ecologically motivated strategies that promote microbiome stability or resilience following a fungicide application.

Fungicide use has become common in conventional agricultural systems. Yet, concerns remain about direct and indirect effects on non-targeted organisms, consequences (i.e., resistance), and negative impacts on the environment or human health [6-8]. The rapid evolution of fungicide resistance in plant and human pathogenic fungal populations can cause devastating epidemics in agricultural ecosystems, with spill-over effects to public health [9-12]. For example, there is substantial concern about the overuse of azole fungicides that have been linked to the resistance of Aspergillus fumigatus to antifungals in human infections [11, 12].
Despite concerns, foliar fungicide applications in maize (Zea mays L.) and soybean (Glycine max L. Merr) are often made without pathogen pressure due to perceived or marketed yield benefits $[13,14]$. A meta-analysis of soybeans demonstrated that foliar fungicide application in the absence of disease increased yield by $2.7 \%$, but applications are less profitable without disease pressure [14]. While fungicides are necessary for crop protection, minimizing non-target effects and unintended consequences is critical in evaluating the sustainability of agricultural production systems.

Studies reporting fungicidal and pesticidal impacts on microbiomes $[15,16]$ have focused on soil and aquatic systems [8] rather than effects on foliage microbes. The two most popular fungicide classes used in agricultural field crops are the sterol demethylation inhibitors (DMIs), otherwise known as triazoles, and quinone outside inhibitors (Qol), or strobilurins. Foliar fungicides for maize and soybean are primarily applied as single or premixed Qol and DMI active ingredients [17]. Qol fungicides inhibit fungal respiration by blocking the quinol oxidation site in the cytochrome $\mathrm{bc}_{1}$ complex in the electron transport chain. DMI fungicides inhibit CYP51 (encoding 14a-demethylase), an important enzyme in the ergosterol biosynthesis pathway of fungi [18]. Both fungicide classes are highly active against many plant pathogens. From the few studies focused on the plant phyllosphere, a consistent non-target effect is detected against phyllosphere yeasts. One study on grapevine microbiomes reported

\footnotetext{
${ }^{1}$ Department of Plant, Soil and Microbial Sciences, Michigan State University, East Lansing, MI 48824, USA. ${ }^{2}$ Department of Microbiology and Molecular Genetics, Michigan State University, East Lansing, MI 48824, USA. ${ }^{3}$ Department of Plant Biology, Michigan State University, East Lansing, MI 48824, USA. ${ }^{4}$ Present address: Department of Entomology and Plant Pathology, Auburn University, Auburn, AL 36849, USA. ${ }^{5}$ These authors contributed equally: Zachary A. Noel, Reid Longley. ${ }^{\circledR e m a i l: ~ b o n i t o @ m s u . e d u ~}$
}

Received: 19 November 2021 Revised: 8 February 2022 Accepted: 10 February 2022

Published online: 23 February 2022 
minimal and transient impacts to the phyllosphere microbiome, including phyllosphere yeast abundance [19]. Similarly, repeated application of broad-spectrum fungicides has been shown through culture-based and culture-independent methods to decrease phyllosphere yeast richness [20-23].

Yeasts that inhabit the phyllosphere are well suited to oligotrophic and dynamic environmental conditions present on leaf surfaces and consequently have been applied for biocontrol of plant pathogens [24]. They are known to produce extracellular polysaccharides and surfactants, which may be necessary for creating or maintaining biofilms [25]. In addition, some phyllosphere yeasts, including species of basidiomycete yeasts in Cryptococcus and Sporidiobolus, produce carotenoid compounds, which have antioxidant properties and may protect the yeasts and other resident microbes from stress in the phyllosphere [26]. Phyllosphere yeast communities have also been linked to pollinator insects by altering floral nectary chemistry, and fungicides can modify this relationship $[27,28]$. However, few studies have addressed the links between phyllosphere yeasts and other phyllosphere residing microorganisms. One study, which did analyze the links between phyllosphere yeasts and bacteria, found evidence that phyllosphere yeasts have direct interactions with bacterial members of the microbiome [29]. While indirect and collective effects of removing single species or groups of species from ecosystems have been proposed in ecological theory since the 1940s and studied in various macroorganism contexts such as conservation biology, disturbance ecology, and food web ecology, such effects are comparatively understudied in microbiome science [30-32]. In microbiomes, network complexity (i.e., linkage density) has been correlated to ecosystem functioning and stability [33, 34]. Consequently, cooccurrence patterns may reveal indirect effects, which may not be seen using other analyses.

Since the US Dust Bowl of the 1930s, soil conservation efforts have led to the steady adoption of minimum or no-till agriculture management systems [35]. Cropping management systems have been demonstrated to impact phyllosphere microbiomes [36, 37]. Crop management's effect on the resilience of foliar fungal communities following fungicides has not been explored, but differing impacts of fungicides in different agricultural managements are probable. In one study performed on soil, agricultural management altered the response of microbial communities to the application of the DMI fungicide tetraconazole [38]. Similarly, a study on wheat demonstrated that crop rotation and wheat variety impacted response to foliar fungicides of various active ingredients, however the crop rotation systems differed between locations, confounding efforts to distinguish fungicide responses in specific rotations from those of location and variety [39]. Longterm experiments circumvent these confounding effects by applying all treatments at a single location.

Here, we characterize effects of foliar fungicides on the maize and soybean leaf and root microbiomes in no-till and conventional plots of the Long-Term Ecological Research (LTER) Main Cropping Systems Experiment at the Kellogg Biological Station (KBS). Our research objectives were three-fold: (1) to determine whether fungicides alter microbial diversity across plant compartments (e.g., leaves or roots), crop species (e.g., maize or soybean), or tillage management (conventional vs. no-till); (2) to identify nontarget and indirect effects of fungicide applications, and (3) determine if crop management alters the resiliency of the microbiome. We hypothesized that fungicides would alter both maize and soybean microbial (fungal and prokaryotic) diversity and network complexity. We predicted that this effect would be most pronounced in the leaves. In addition, given that plant microbiomes have been shown to differ under the two tillage management systems [37], we hypothesize that the response and recovery of plant microbiomes following fungicides would also differ. This LTER site allows for a novel approach by eliminating any differences caused by location bias and assessing the effect of fungicide application under long-term agricultural management. We apply a novel microbiome network analysis approach to determine the impact fungicides have on prokaryote-fungal co-occurrences in the plant microbiome. Finally, we used random forest models to predict prokaryote taxa responsive to altered fungal diversity demonstrating the possible indirect effects of fungicides.

\section{MATERIALS AND METHODS}

\section{Sample site and management systems}

Samples were collected from the no-till and conventional tillage management treatments of the main cropping experiment at Michigan State University's Kellogg Biological Station (KBS) Long Term Ecological Research (LTER) site which follows a maize (Zea mays L.), soybean (Glycine max L. Merr), and winter wheat (Triticum aestivum L.) rotation. The site contains six one-hectare replicate plots of no-till or conventional tillage plots consistently managed since 1989 [40]. Fungicide micro-plots (3.05 m wide $\times 6.10 \mathrm{~m}$ long) were established within four replicate plots in no-till and conventional tillage treatments. Control samples were taken from microplots of the same size directly next to the fungicide micro-plots. Samples were taken from the middle of plots to minimize the effect of any spray drift. Fungicide applications of Headline with the Qol active ingredient pyraclostrobin (2017) and Delaro with the combination of the DMI prothioconazole and Qol trifloxystrobin active ingredients (2018) were performed at recommended label rates and are described in detail in the supplementary methods.

\section{Sample collection}

In 2017, maize leaf and root samples were collected at three time points. The first sampling occurred before the fungicide application on 26 June 2017 (V6 growth stage), the second was 9-days post fungicide (dpf) (V8 growth stage), and the final sampling was 35-dpf (V15 growth stage). Leaves and roots from three plants from four replicate control or adjacent fungicide treated plots of each no-till, and conventional management were sampled at each time point. In 2018, soybean leaves were sampled at three time points the first occurred before fungicide spray on 3 August 2018 (R3 growth stage), the second occurred 13-dpf (R4 growth stage), and the final occurred 33-dpf (R6 growth stage) [41].

Sampling and DNA extractions were performed as described previously $[37,42]$. In addition, amplicon sequencing library preparation, bioinformatic sequence processing, and preprocessing before statistical analyses were performed as previously described [37, 43, 44]. Details for these processes are described in the Supplementary Methods.

\section{Statistical analysis}

Differences in fungal and prokaryotic community composition were tested through permutational multivariate analysis of variance (PERMANOVA) with the 'adonis2' function on Bray-Curtis distances in the R package vegan [45]. Variation in multivariate dispersion was tested with the 'betadisper' function in vegan. More specific hypotheses were tested based on constrained analysis of principal coordinates (CAP) [46] using the 'capscale' function in vegan. Differentially abundant taxa resulting from fungicide application were identified by comparing fungicide treated plots to control plots through an analysis of the composition of microbiomes (ANCOM v 2.1) [47]. For differential abundance analysis, fungal OTUs (fOTU, hereafter) with a mean relative abundance less than $10^{-5}$ and fOTUs with zeros present in $95 \%$ samples were discarded from the analysis to avoid detecting fOTUs as significantly different based on stochasticity. In addition, fOTUs that were never present in fungicide treated plots were not included. Fungal OTUs were determined to be significant if the $W$ value was greater than $70 \%$ of the taxa tested based on Wilcoxon ranked sum test between additive log-ratio transformed data and a BenjaminiHochbergj adjusted $P$ value $(a=0.05)$ [47]. Recovered taxa (i.e., transient effects) were defined as fOTUs that were significantly less abundant in the first sampling following fungicide treatment but were not significantly less abundant from non-disturbed plots, after 33- or 34-dpf, for soybean or maize, respectively. Non-recovered taxa were defined as those fOTUs with significantly altered abundance following fungicide application and remained significantly altered for the remainder of the sampling. In addition, a portion of non-recovered taxa was also locally extinct, which were defined as taxa present before fungicide application but having zero relative abundance following fungicide application in fungicide treated plots through the remainder of the sampling while being present in the 
control plots. Finally, taxa that did not have significantly altered abundance following fungicide application but then had significantly different abundance at a later sampling point (i.e., 33- or 34-dpf) were defined as indirect effects.

The core phyllosphere fungal and prokaryotic taxa from the non-fungicide disturbed no-till or conventional plots were identified based on each abundance and occupancy across space and time. Taxa that contributed to the last $2 \%$ increase in Bray-Curtis distances were defined as the core [48].

We built random forest regression models to test the effect of altered prokaryote abundance through fungal diversity by using prokaryote abundances to predict fungal diversity. Random forest models were generated with the 'randomForest' function in the randomForest $\mathrm{R}$ package [49]. To remove redundant features and avoid overfitting models, we removed redundant OTUs with the 'Boruta' function in the package Boruta [50]. The method performs a top-down search for relevant OTUs by comparing the importance of the original OTUs from those selected at random. Models were tuned to achieve the lowest stable out-of-bag (OOB) error estimate possible, and the best mtry value (number of OTUs sampled at random in the entire pool for each tree at each split) was selected using the 'tuneRF' function in randomForest $\mathrm{R}$ package.

Network analysis was conducted on soybean and maize leaf samples to estimate co-occurrences among prokaryotic and fungal OTUs in each host and determine whether fungicides altered fungal-prokaryotic co-occurrences and network complexity (i.e., linkage density). For network analyses, soybean and maize fungal and prokaryotic OTU tables were filtered to exclude taxa with mean relative abundance below $10^{-5}$. A co-occurrence meta-matrix was estimated using the Meinshausen and Bühlmann algorithm within the SpiecEasi R package with the 'nlambda' set to 100 and with 'lambda.min.ratio' set to $10^{-2}$ [51]. From this meta-matrix subnetworks were created from taxa present within each sample. Then, network complexity was calculated for each subnetwork. The contribution of the Bulleribasidiaceae to network complexity was assessed by examining the change in the cumulative edge weights across subnetworks with prokaryotic genera. Bulleribasidiaceae were selected for further analysis because they represent an off-target group of fungi that was substantially impacted by fungicide application.

\section{RESULTS}

\section{General sequencing results}

The final fungal OTU table contained 20,844,912 ITS1 reads across 554 samples, including 5,315 fOTUs after filtering 36 contaminant fOTUs detected in negative controls. The median read depth was 30,370 ITS1 reads per sample. Prokaryotes contributed 29,691,681 total reads across 555 samples with a median read depth of 47,590 reads per sample. A total of 14,291 prokaryote OTUs (pOTU, hereafter) were defined after filtering 55 contaminant pOTUs detected in the negative controls. Rarefaction curves verified that the median read depth adequately sampled the diversity present (Fig. S1).

\section{Fungicides alter the maize and soybean leaf fungal richness} Following fungicide application, the richness of maize and soybean leaf fungal communities was significantly reduced compared to control plots across managements and crops $(P<$ 0.05 ) except for in no-till maize samples. (Fig. S2). This effect was most pronounced for Dothideomycetes (target) and Tremellomycetes (non-target). However, in other fungal classes such as Sordariomycetes, there was no significant difference in richness between control and fungicide treated samples following fungicide applications. There were no significant differences in fungal richness between fungicide and control plots amongst the assessed fungal classes or in overall richness in the roots of either crop (Fig. S3). Among prokaryotes, there were no consistent differences between control and fungicide samples in the leaves or roots of either crop (Fig. S4).

\section{Fungicides alter the maize and soybean leaf fungal community composition}

Fungal and prokaryote community composition varied significantly due to timepoint (i.e., dpf) and crop management in maize and soybean leaves and roots (Table S4; Table S5). Notably, before fungicides were sprayed, crop management was shown to have a significant effect on the maize and soybean phyllosphere fungal and prokaryotic communities (maize leaf fungi $\mathrm{R}^{2}=0.050, P=$ 0.001 ; maize leaf prokaryotes $R^{2}=0.038, P=0.005$; soybean leaf fungi $R^{2}=0.058, P=0.020$; soybean leaf prokaryotes $R^{2}=0.046$, $P=0.049$ ). Furthermore, fungal and prokaryotic phyllosphere community compositions in control and treatment plots were indistinguishable from each other prior to applying fungicide treatments (maize leaf fungi $R^{2}=0.032, P=0.051$; maize leaf prokaryotes $R^{2}=0.023, P=0.418$; soybean leaf fungi $R^{2}=0.018$, $P=0.483$; soybean leaf prokaryotes $R^{2}=0.035, P=0.128$ ). Despite this, changes to the fungal phyllosphere composition by fungicide treatments differed depending on management (fungicidemanagement interaction) only in the soybean, but not in the maize leaves (maize leaf fungi 9-dpf $R^{2}=0.012, P=0.916$; soybean leaf fungi 13 -dpf $R^{2}=0.041, P=0.017$; soybean leaf fungi 33-dpf $\left.R^{2}=0.039, P=0.015\right)$. There was no substantial evidence that fungicides altered the composition of phyllosphere prokaryote communities, prokaryote root communities, or fungal root communities. Therefore, the variance explained due to the fungicide disturbance was examined for fungal phyllosphere communities before and after fungicide exposure while partitioning out the variation due to crop management by a constrained analysis of principal coordinates (CAP) (Fig. 1).

For soybean leaves, no significant differences were observed prior to fungicide application $(P=0.51)$, but fungicide treatment had a significant effect on fungal leaf composition after fungicides were applied (13-dpf $12 \%$ variation $P<0.001 ; 33-d p f 11 \%$ variation $P<0.001$ ) (Fig. 1a-c). Similarly, the effect of fungicide disturbance on maize leaf fungal composition was not observed before fungicides were applied $(P=0.075)$ (Fig. 1d). However, unlike soybean, there was no evidence the fungicide altered fungal composition longer than nine days $(9-\mathrm{dpf} 8 \%$ variation $P<0.001$; 34-dpf $3 \%$ variation $P=0.078$ ) (Fig. 1e, f). The non-significant beta dispersion tests across tillage management at 9-dpf or 34-dpf for maize (9-dpf conventional $P=0.369$, no-till $P=0.631 ; 34-\mathrm{dpf}$ conventional $P=0.364$, no-till $P=0.662$ ) and 13- and 33-dpf (13dpf conventional $P=0.742$, conventional $P=0.866$; 33-dpf conventional $P=0.335$, no-till $P=0.123$ ) for soybean, indicate that the effects of fungicide on fungal leaf composition are likely due to true differences in community composition rather than group dispersions (Table S6).

\section{Fungicidal effects on network properties depend on crop management}

In soybean under conventional and no-till management, network complexity was not significantly different before fungicide application (conventional $P=0.13$; no-till $P=0.93$ ), but was significantly lower than control plots 13-dpf (conventional $P<$ 0.001 ; no-till $P=0.01$ ) (Fig. 2a). However, after one month, the soybean no-till network complexity had recovered $(P=0.12)$, whereas the conventional treatment was still significantly lower compared to the non-sprayed control plots ( $P=0.002$ ) (Fig. 2a). The loss in network complexity can partially be explained by a reduction in the number of nodes (i.e., OTUs) since the average number of nodes per network also followed this same trend (Fig. 2b). Fungicide disturbance was followed by the loss of network complexity mainly through node loss but crops and crop management impacted network properties under fungicide disturbance. These same effects were not observed in maize (Fig. S5). To investigate these trends more closely, we investigated the specific fungal taxa affected through differential abundance analysis.

\section{Identification of fungicide-affected fOTUs}

To determine which fungal taxa were significantly affected by fungicide application, a differential abundance analysis was 
Control $\bigcirc$ Fungicide $\bigcirc$ Conv. $\square$ No-till

(a) Soybean Leaf - 0-dpf

$2 \%$ of total variation $(\mathrm{Cl}=1 \%, 4 \%) ; \mathrm{P}=0.51$

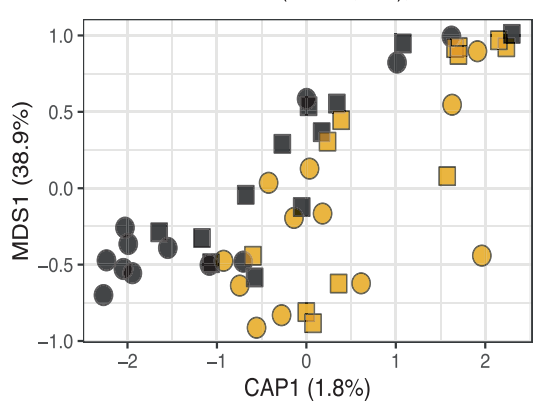

(d) Maize Leaf - 0-dpf

$3 \%$ of total variation $(\mathrm{Cl}=2 \%, 5 \%) ; \mathrm{P}=0.075$

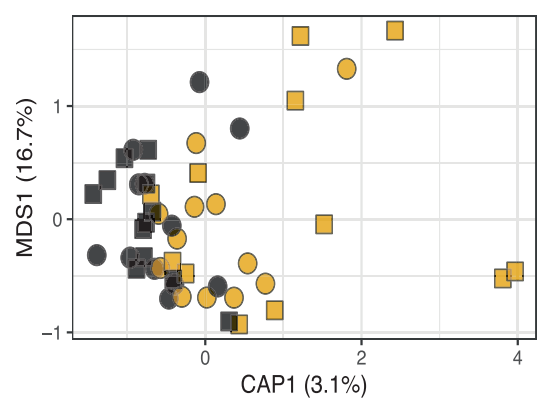

(b) Soybean Leaf - 13-dpf

$12 \%$ of total variation $(\mathrm{Cl}=6 \%, 22 \%) ; \mathrm{P}<0.001$

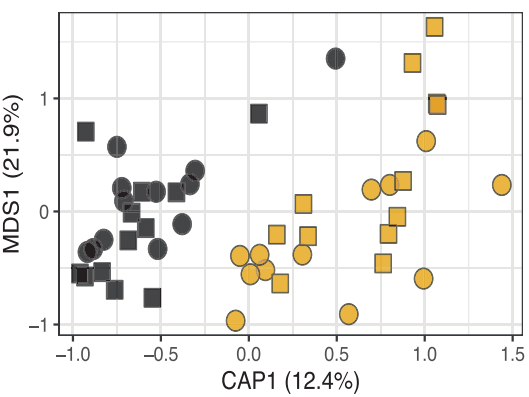

(e) Maize Leaf - 9-dpf

$8 \%$ of total variation $(\mathrm{Cl}=5 \%, 13 \%) ; \mathrm{P}<0.001$

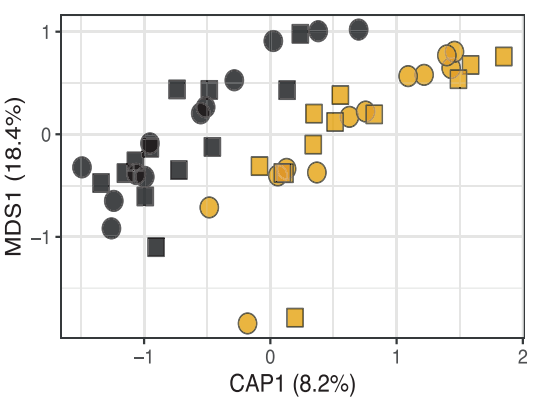

(c) Soybean Leaf - 33-dpf

$11 \%$ of total variation $(\mathrm{Cl}=6 \%, 18 \%) ; \mathrm{P}<0.001$

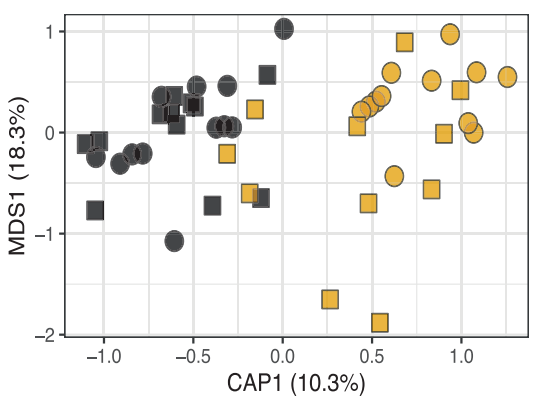

(f) Maize Leaf - 34-dpf

$3 \%$ of total variation $(\mathrm{Cl}=2 \%, 6 \%) ; \mathrm{P}=0.078$

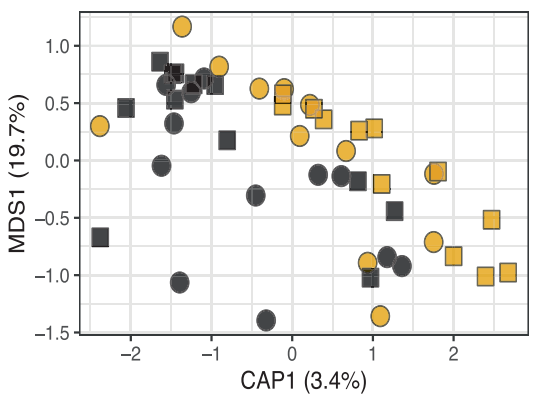

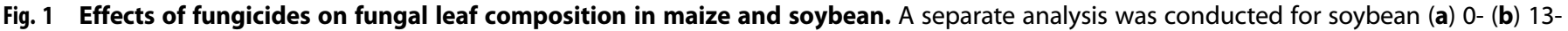

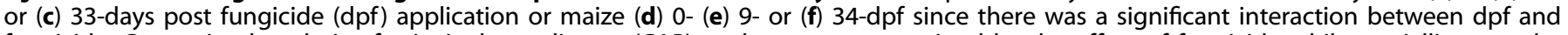

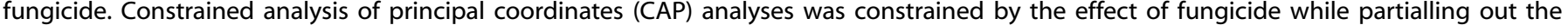

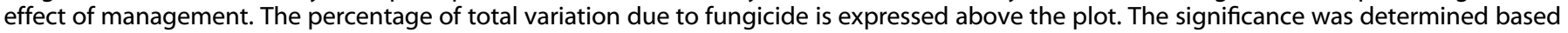
on 1000 permutations.

Control to Fungicide

(a)

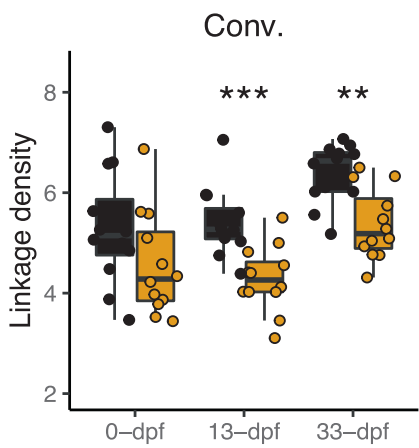

Soybean

No-till

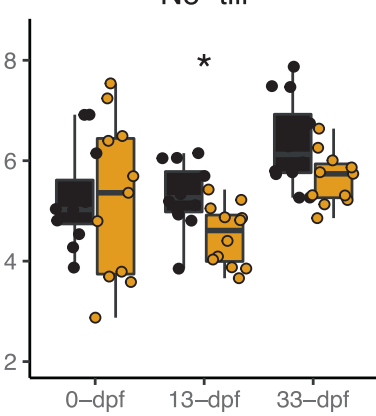

(b)

Soybean

Soybean

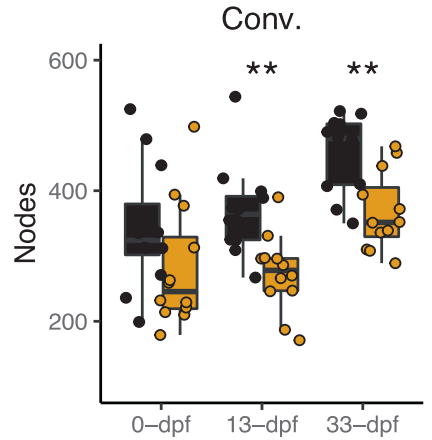

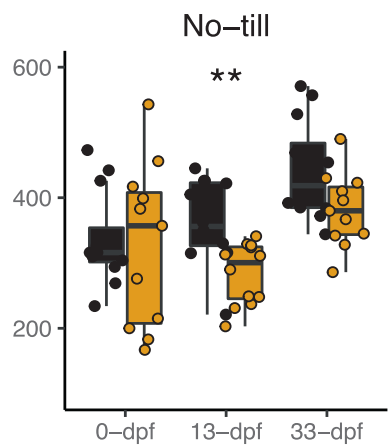

Fig. 2 Fungicides alter soybean network complexity. A microbial co-occurrence network was constructed using taxa with a mean relative abundance greater than $1^{-5}$ and present in greater than $5 \%$ of samples. Subnetworks were generated for each sample based on the OTUs present within those samples, and each point represents a subnetwork. a Network complexity (i.e., linkage density) and (b) number of edges were then calculated for each subnetwork. Comparisons are based on Wilcox ranked sign tests for soybean conventional management and no-till. An asterisks indicate the level of significance; ${ }^{*}=p \leq 0.05,{ }^{* *}=p \leq 0.01,{ }^{* * *}=p \leq 0.001$. Comparisons for maize are shown in Fig. S5.

conducted with ANCOM (Table S7). In total, the abundance of 238 unique fOTUs representing 21 fungal classes was altered by fungicide treatments across the two crops. Ascomycota (52.9\%) and Basidiomycota (43.3\%) fOTUs made up $96.2 \%$ of the differentially abundant fOTUs. Within Ascomycota, the Dothidiomycetes (28.6\%) and Sordariomycetes (9.66\%) accounted for the largest percentage of fOTUs that were differentially abundant following fungicide treatment (Fig. 3a). These fungi may be expected since many foliar plant pathogens fall within these classes of fungi, and fungicides typically target these pathogen groups. Unexpectedly, a large percentage of fOTUs that were differentially abundant included non-target dimorphic clades of fungi that commonly exist as yeasts such as Agaricostilbomycetes, Cystobasidiomycetes, Exobasidiomycetes, Microbotryomycetes, Spiculogleomycetes, Taphrinomycetes, and Tremellomycetes. A total of 83 fOTUs across these classes were significantly different in abundance following the fungicide application in maize or soybean (Fig. 3a). Notably, Tremellomycetes made up the second- 
(a) Fungi

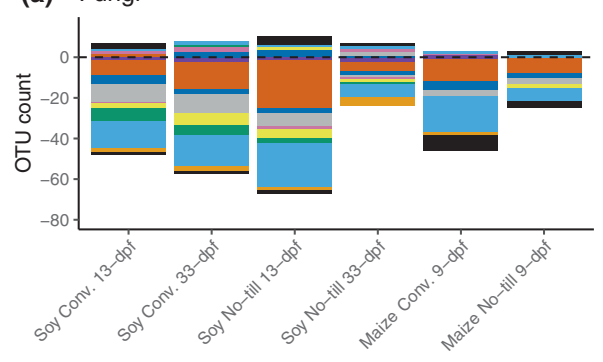

(b) Fungi - Soybean

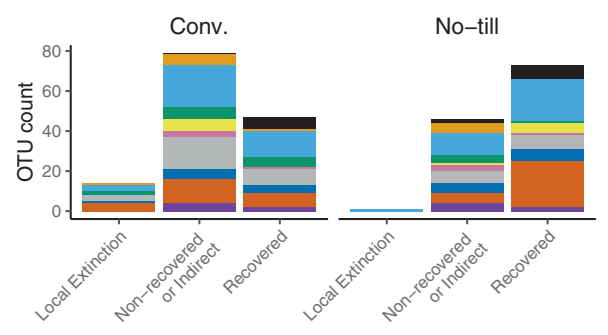

(c) Tremellomycetes

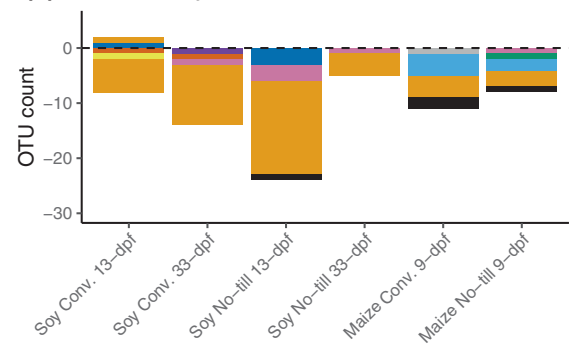

(d) Tremellomycetes - Soybean

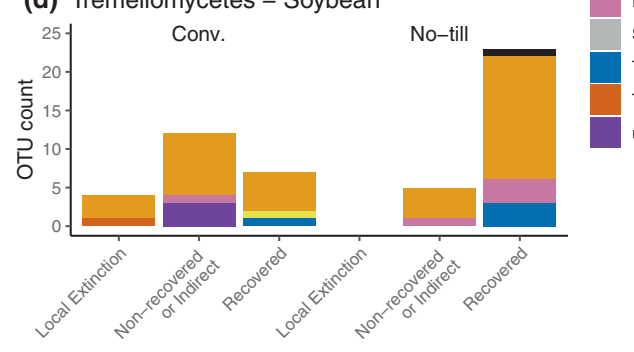

(e) Soybean

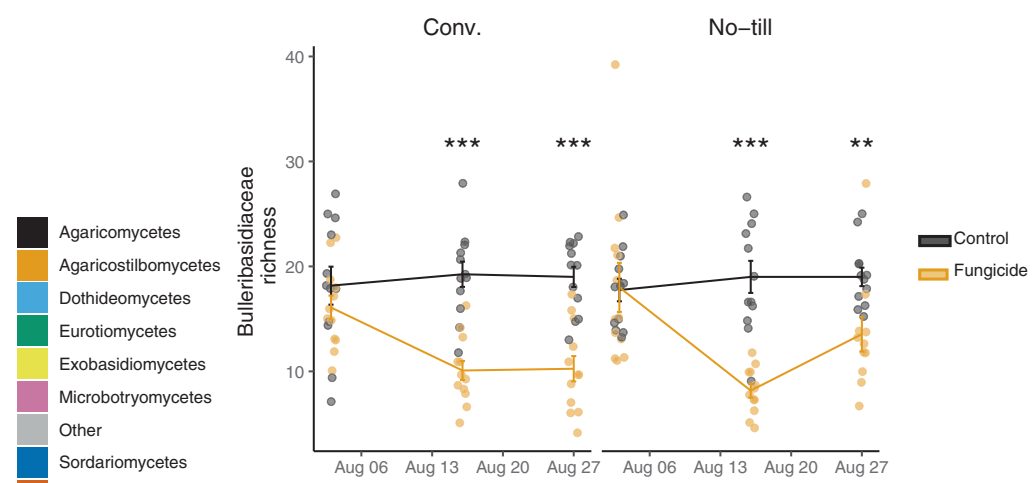

(f) Maize

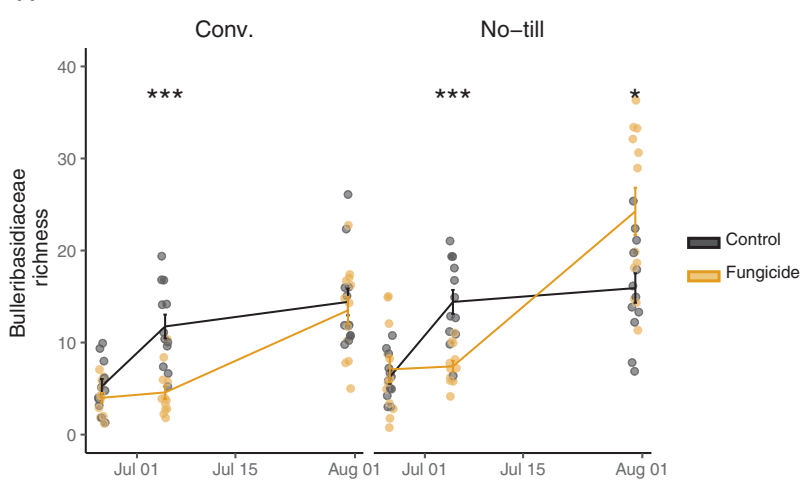

(g) Soybean

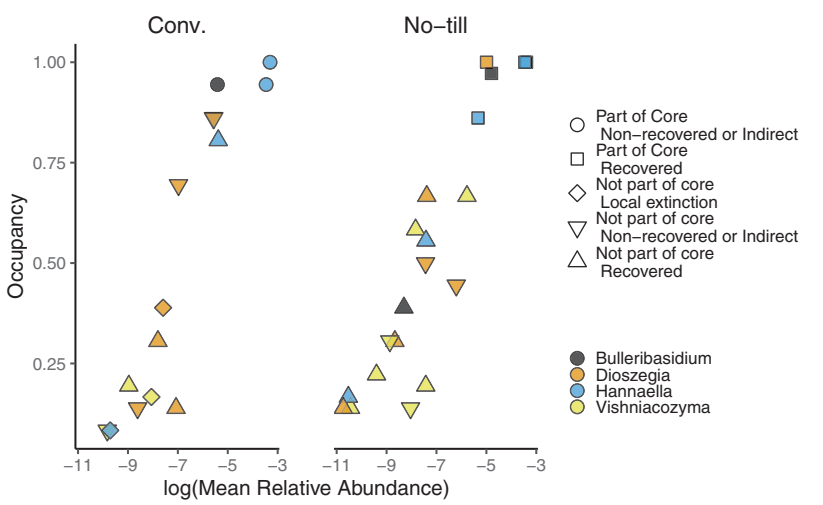

Fig. 3 Management scheme alters the recovery dynamics of phyllosphere fungi following fungicide treatment. The composition of fungal operational taxonomic units (OTUs) that were significantly different in abundance, as indicated with analysis of compositions of microbiomes (ANCOM) analysis $(n=12)$. a Composition of fOTUs whose abundance was significantly different following a fungicide disturbance. Bars below zero indicate the fOTU decreased in abundance, whereas bars above zero indicate the fOTU increased in abundance. $\mathbf{b}$ Recovery of fungi in soybean leaf samples in conventional (conv.) or no-till management. All fungi recovered in maize leaf samples. c Composition of fOTUs within the Tremellomycetes whose abundance was significantly altered following a fungicide disturbance. $\mathbf{d}$ Recovery dynamics of Tremellomycetes fOTUs following a fungicide disturbance in conv. or no-till. All Tremellomycete fungi recovered in maize. e Soybean or (f) maize plots subjected to a fungicide treatment compared to non-sprayed control plots. g Abundance occupancy relationship with the recovery dynamics of the Bulleribasidiaceae fOTUs significantly affected by the fungicide treatment. All Bulleribasidiaceae fOTUs recovered in maize. A full list of core fungi and prokaryotes for soybean or maize leaves are found in Table S8. An asterisks indicate the level of significance; ${ }^{*}=p \leq 0.05,{ }^{* *}=$ $p \leq 0.01,{ }^{* * *}=p \leq 0.001$.

largest class (42 fOTUs, $17.6 \%$ ) of differentially abundant fungi. Of the Tremellomycetes, $57.1 \%$ were concentrated within the Bulleribasidiaceae, accounting for 24 fOTUs that were differentially abundant compared to non-sprayed control. Twenty-three of the Bulleribasidiaceae significantly decreased in abundance. However, not all yeast fOTUs decreased in abundance. For example, Bulleromyces albus fOTU10 increased in relative abundance 4.25 times in soybean conventional management 13-dpf but was not significantly different than the control after 33-dpf. In contrast, two Sporobolomyces fOTUs (fOTU66 and fOTU94) increased relative abundance following fungicide application in soybean and remained significantly (7 times) higher in fungicide treated plots than in control plots 33-dpf. Sporobolomyces patagonicus fOTU94 was 4.38 times more abundant in the fungicide treated plots than the control 13-dpf in the conventional management and remained significantly higher in fungicide sprayed plots (9.06 times) compared to the control after 33-dpf. Sporobolomyces roseus fOTU66 was 15 times more abundant in the conventionally 


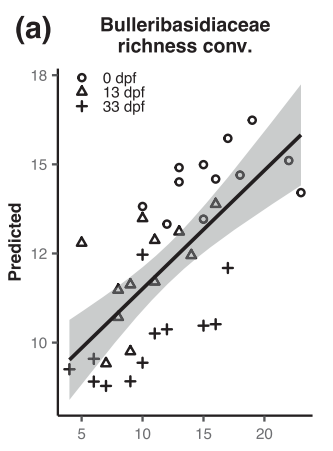

Observed

(c) Bulleribasidiaceae

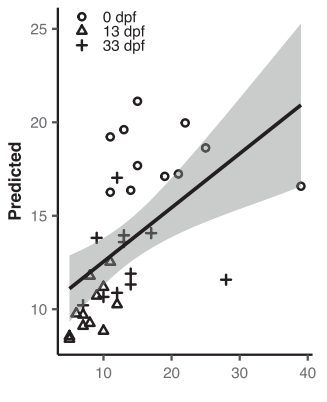

Observed (b)
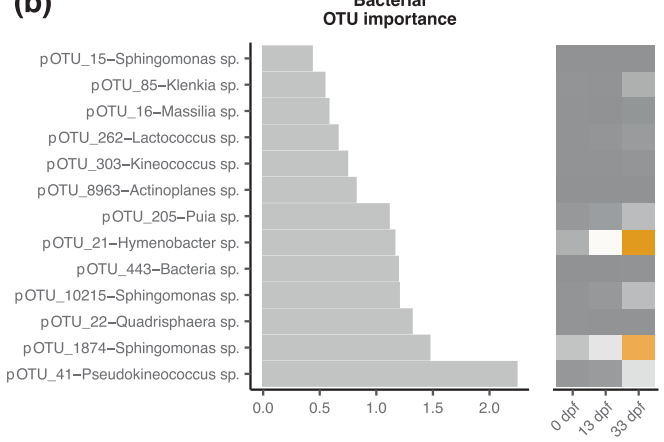

MSE Increase

(d)

Bacterial

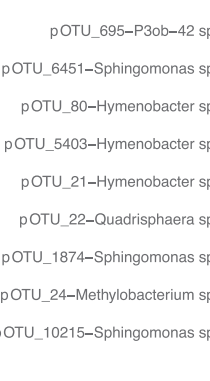

terium sp.<smiles>CCC</smiles>

Fungicide Control

(e)
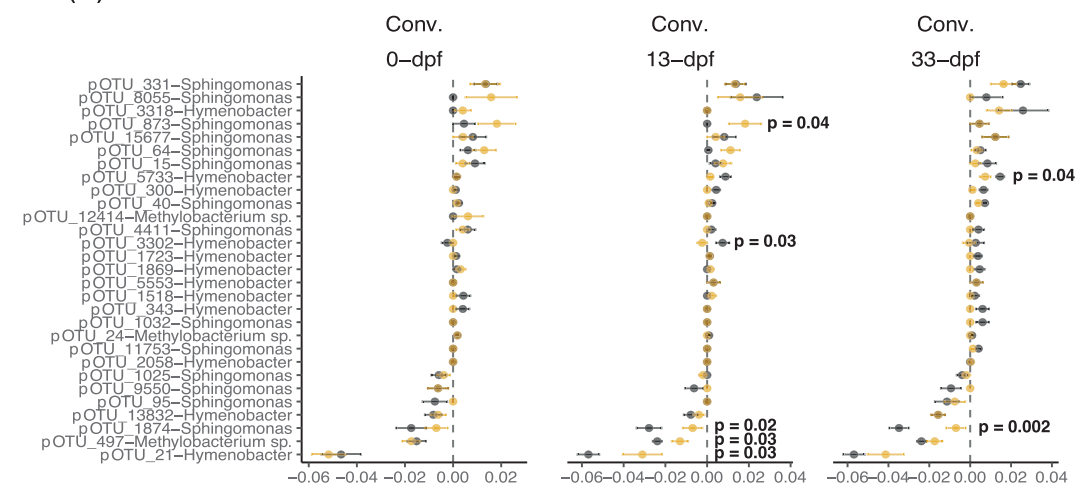

(f)
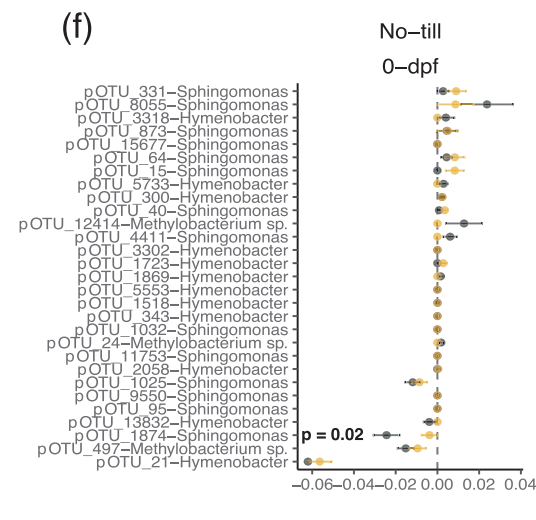

No-till

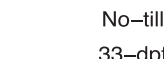

Read No.

10000

5000

0 
managed fungicide treated plots 33-dpf. This same increase in Sporobolomyces abundance was not observed in maize.

\section{Resilience of the core mycobiome and local extinctions of accessory members}

Many of the fOTUs affected by a fungicide application were also part of the core phyllosphere taxa (Table S8). In conventionally managed soybean plots, 22 fOTUs were determined to be core fungal phyllosphere taxa, and the abundances of five of these core members (fOTU 6 Mycosphaerella sp., fOTU 10 Tremellales, fOTU 34 Hannaella sp., fOTU 13 Hannaella sp., and fOTU 643 Tilletiopsis sp.) were significantly different following fungicide application. Hannaella sp. (fOTU 34), Hannaella sp. (fOTU 13), and Tilletiopsis sp. (fOTU 643) were also part of the 43 core members of the no-till soybean phyllosphere affected by fungicide application. Of the 40 core members of the conventionally managed maize phyllosphere, the abundance of four Tremellomycetes fOTUs and one unidentified fungal taxon (fOTU 116) were significantly different following fungicide application. These included three yeast taxa that were not members of the soybean core, which included two fOTUs in the genus Filobasidium (fOTU 82 Filobasidium oeirense, and fOTU 97 Filobasidium sp.), one Bullera crocea (fOTU 65), and Vishniacozyma globispora (fOTU 83). Two of these fOTUs (fOTU 97 Filobasidium and fOTU 65 Bullera crocea) were also core members of the maize phyllosphere in the no-till management that were significantly altered by the fungicide.

None of the core members of the phyllosphere taxa became locally extinct following fungicide application in the core microbiome of either crop or tillage management. However, among the taxa whose abundance was significantly altered by the fungicide application in soybean, the no-till management had a 61 $\%$ recovery compared to the $34 \%$ recovery in the conventional tilled soybean (Fig. 3b; Table S9). Fourteen fungal OTUs became locally extinct following fungicide application in soybean with conventional tillage compared to one in the no-till plots (Fig. 3b). Among the Tremellomycetes fOTUS whose abundances were significantly impacted by fungicide applications, the majority were Bulleribasidiaceae (Fig. 3c). Eighty-two percent of affected Bulleribasidiaceae recovered in no-till managed soybean compared to the $30 \%$ of the conventionally tilled plots. No Bulleribasidiaceae taxa became locally extinct in the no-till plots; in contrast, three Bulleribasidiaceae fOTUs were never observed again following fungicide application in the conventional tilled management (Fig. 3d). The trend of increased recovery was also evident in Bulleribasidiaceae richness on the last sampling for maize (33-dpf) and soybean (34-dpf) no-till samples (Fig. 3e, f). Bulleribasidiaceae in the core of conventional tillage did not fully recover within the study period (Fig. $3 \mathrm{~g}$ ). In addition, the Bulleribasidiaceae in the conventional tillage management that were locally extinct following fungicide disturbance occupied less than $50 \%$ samples in non-sprayed plots indicating that local extinctions caused by fungicides affect the rare, non-core members of the community (Fig. $3 \mathrm{~g}$ ). No local extinctions among fungal taxa were detected in maize fungicide treated plots; all impacted taxa recovered.

\section{Indirect effects of fungicides on prokaryotes mediated through yeast}

Random forest models based on prokaryotic abundance on soybean leaves sprayed with fungicides explained a significant amount of variance $(P<0.001)$ in Bulleribasidiaceae richness in the no-till $\left(28.70 \% ; R^{2}=0.25\right)$ and conventional tillage $\left(43.47 \%\right.$; $R^{2}=$ 0.44 ) management (Fig. $4 a, c)$. We then extracted the set of OTUs that were the most important for maintaining the model's accuracy in fungicide treated plots. However, there was no evidence $(P \geq 0.05)$ those same taxa were able to predict Bulleribasidiaceae richness in control samples indicating the unique effect of the fungicide (Fig. S6). OTUs classified as
Sphingomonas, Methylobacterium, and Hymenobacter were the most important for predicting fungal richness in the no-till management (Fig. 4c, d). Many taxa from the same genera were important in predicting Bulleribasidiaceae richness in the conventional tillage management, including the Sphingomonas and Hymenobacter genera (Fig. 4a, b). However, other genera were unique by management type, including Methylobacterium for the no-till management and Pseudokineococcus and Kineococcus in the conventional tillage management.

Prokaryote OTUs that were important for random forest model accuracy increased in abundance in fungicide treated plots as a response to altering Bulleribasidiaceae diversity and were negatively co-associated with Bulleribasidiaceae (Fig. 4b, d, e, f). For example, pOTU21 Hymenobacter and pOTU1874 Sphingomonas abundance increased as Bulleribasidiaceae richness decreased and was negatively co-associated with the Bulleribasidiaceae (Fig. 4b, e, f). In addition, the cumulative edge weight between pOTU21 Hymenobacter, pOTU1874 Sphingomonas, and Bulleribasidiaceae significantly changed when sprayed with fungicides in the conventional tillage management, but not always in the notill management, indicating that an alteration in Bulleribasidiaceae diversity can indirectly influence the co-occurrence between fungi and bacteria in different crop management schemes. However, not all co-occurrences between the Bulleribasidiaceae and prokaryotes were negative, indicating that positive cooccurrences between prokaryotes and fungi in the phyllosphere may shift as well (Fig. 4e, f).

\section{DISCUSSION}

To our knowledge, this is the first study to assess the effect of fungicide-imposed disturbance and resiliency under different agricultural management systems. We found that fungicide applications had a substantial effect on target and non-target fungal phyllosphere communities, minor indirect effect on prokaryotic communities in the phyllosphere, and no direct effects on fungal or prokaryotic communities of roots. Soil fungi and prokaryotes were also identified in soybeans, where there was no evidence of fungicidal effects (data not shown). Leveraging the KBS LTER site allowed the direct comparison of long-term crop management impacts to the microbiome without confounding location. Our data demonstrate that the resilience of phyllosphere microbiome depends on the cropping management system, with a greater recovery in the abundance of affected phyllosphere microbiota in long-term no-till compared to annually conventional tilled management. Among the most important results was the commonality in the fungal taxa affected by fungicide treatments. In maize and soybean, fungi in Dothidiomycetes (target) and Tremellomycetes (non-target) decreased in abundance following fungicide applications, raising questions on the role of Tremellomycete yeasts; specifically, the Bulleribasidiaceae in phyllosphere microbiomes, and the effects of fungicide use in the absence of disease pressure.

This study observed reductions and local extinctions of yeasts following fungicide application, which may lead to unintended consequences for the host plant. Phyllosphere yeast communities have received less attention in the literature than prokaryote communities [52]. The three Bulleribasidiaceae genera observed in this study were Hannaella, Dioszengia, and Vishniacozyma. Dioszengia, and Hannaella have been demonstrated to produce the plant growth-promoting hormone indole acetic acid (IAA), similar to many plant growth-promoting phyllosphere prokaryotes $[53,54]$. In comparison, Vishniacozyma yeasts have remained understudied but have been isolated from maize kernels [55]. In addition, Dioszegia has been identified as a hub taxon important in maintaining fungal-prokaryote interactions by altering prokaryote diversity in the phyllosphere microbiome of Arabidopsis $[29,53]$. As observed in this study, in the absence of 
disease pressure, fungicide applications may affect populations of beneficial microbes. However, adverse impacts would be expected to be outweighed if the fungicide mitigates the disease, which will be tested in future experiments.

Here, we show for the first time that fungicidal impacts on crop microbiomes are dependent on management, addressing a knowledge gap that previous studies were unable to address specifically $[20,21,39]$. A higher proportion of fOTUs altered by fungicide application in the no-till management system showed improved resilience within the study period, which may be explained by the differences in microbial communities present in the phyllosphere of each management before fungicide applications, as has been demonstrated previously at the KBS LTER site $[36,37]$. A previous study from the KBS LTER site demonstrated that aerially dispersed yeasts are enriched in the phyllosphere, but also found in lower abundance in belowground plant organs [42]. Crop residue from previous seasons can harbor fungi that may act as a source to repopulate the phyllosphere following a disturbance like the phenomenon of pathogens transferring from residues [56]. Yeasts that inhabit the phyllosphere are primarily known to disperse through ballistosporic aerial dispersal, and the reassembly of leaves following fungicides may rely heavily on this spore dispersal mechanism. However, not all yeast taxa in the Bulleribasidiaceae have been observed to form ballistocondia in culture [57], leaving arguably less efficient means of dispersal from insects or through wind and rain $[58,59]$. Locally extinct taxa were not part of the core microbiome regardless of tillage management system or spore dispersal mechanism, demonstrating a tight relationship between abundance-occupancy and disturbance. These results indicate that microbiome resilience is improved in no-till crop management, which informs discussion of managing crops for resilience, and demonstrates a potential ecosystem service provided by no-till agriculture in addition to improved nutrient cycling or preservation of habitats for microorganisms and mesofauna [60].

Fungicide applications affected soybean and maize phyllosphere communities differently. These differences may be due to crop, planting year, or fungicide regime. The effect of fungicide was likely reduced in the final sampling of maize due to sampling of new leaves that were not directly sprayed with fungicides, indicating that any effect would have been through systemic activity of the fungicide 34 days after. This is unlikely since pyraclostrobin is not easily xylem mobile and mainly works as a translaminar local penetrant [61]. Another critical difference is that the Delaro fungicide applied to soybeans in 2018 has two modes of action. Application of fungicides having two different modes of action has been shown to have a more significant effect on fungal community composition than a single mode of action in cereal crops [21]. Although the impact of fungicides varied in magnitude between the two crops, the commonality of off-target impacted taxa between crops and fungicides demonstrates that multiple fungicide products on different crops consistently reduce these taxa. This information can be used to inform decisions on the use of fungicides under low pathogen pressure across crops and cropping systems.

Recovery of network complexity is one measure of microbiome resilience. We show that network complexity decreased significantly in the soybean phyllosphere following fungicide treatment. Despite similar affected taxa, the effect of fungicides on maize was moderate compared to soybean, which saw a reduction in network complexity and local extinctions of some taxa. Therefore, we focused more on fungicidal effects to soybean rather than maize. Other studies have demonstrated that agricultural management alters network complexity. However, the functional consequences of these changes were not directly assessed $[62,63]$. In soils, it has been demonstrated that increases in network complexity are positively correlated with various ecosystem functions and increases in the number of unique functions and functional redundancy [34]. The functional consequences of decreases in network complexity remain unexplored in the phyllosphere microbiome. They may provide the rationale for chemical application decisions or novel microbial-based treatments to replace lost taxa.

Notably, fungicide application altered co-occurrences between phyllosphere fungi and prokaryotes, demonstrating the indirect effects of fungicide applications through the loss in the diversity of Bulleribasidiaceae. In support of random forest results, many of the same prokaryotes identified from networks as having changes in cumulative mean edge weight were identified by random forest as predicting Bulleribasidiaceae richness. Disturbance can change cooperation/competition dynamics, and a high level of disturbance can reduce cooperation [64, 65]. In our study, the cumulative mean edge weight between most phyllosphere prokaryotes and Bulleribasidiaceae became more positive, indicating fewer negative associations between particular bacteria and the Bulleribasidiaceae. However, there were exceptions where cumulative edge weights, positive before spray, became neutral following fungicide application likely due to the disappearance of some fungal taxa from samples, and therefore the disappearance of any associations. Loss of negative correlations may also be due to reduced competition between phyllosphere prokaryotes and Bulleribasidiaceae as more niche space is available to phyllosphere prokaryotes following fungicide application.

Shifts in correlations between Bulleribasidiaceae and phyllosphere prokaryotes are of interest due to the unique physiology of many phyllosphere prokaryotes as it relates to plant health. Methylobacterium spp. have been demonstrated to be abundant in plants' phyllosphere and have the genes to produce plant growth-promoting auxins and UVA-absorbing compounds $[66,67]$. Hymenobacter sp., Methylobacterium sp., and Sphingomonas sp. are core phyllosphere members in switchgrass [68] and are highly abundant in the Arabidopsis phyllosphere [69].

A comprehensive view of the phyllosphere organisms is needed to understand microbiome functioning and plant health. This research demonstrates that foliar fungicide treatments alter phyllosphere microbiomes in maize and soybean, and nontarget Bulleribasidiaceae yeasts were negatively impacted in both crops. Microbiome complexity was altered partially by decreasing co-occurrence between Bulleribasidiaceae yeasts and dominant phyllosphere prokaryote taxa, demonstrating indirect effects of fungicide applications mediated through the presence of these yeasts. Further, these data support our hypothesis that the recovery of the phyllosphere microbiome differed by tilling management. Together, these results improve our understanding of fungicide impacts on crop microbiomes and their recovery in different managements and inform their rational use to maintain efficacy and intended impacts across different cropping systems.

\section{DATA AVAILABILITY}

Raw sequences for soybean microbial communities used to create figures and tables in this study are available in the NCBI SRA database under the following accession numbers: PRJNA603199 and PRJNA603207. Sequences produced on the same Miseq runs and used to remove contaminants are available in PRJNA603147. Raw sequences for maize microbial communities are available under the following accession numbers: PRJNA739465 and PRJNA739759. Code to generate figures and tables are located on GitHub at https://github.com/noelzach/FungicidePulseDisturbance

\section{REFERENCES}

1. Rykiel EJ. Towards a definition of ecological disturbance. Austral Ecology. 1985;10:361-5.

2. Glasby TM, Underwood AJ. Sampling to differentiate between pulse and press perturbations. Environ Monit. Assess. 1996;42:241-52.

3. Sullivan TP, Sullivan DS. Vegetation management and ecosystem disturbance: impact of glyphosate herbicide on plant and animal diversity in terrestrial systems. Environ Rev. 2003;11:37-59.

4. Landers TF, Cohen B, Wittum TE, Larson EL. A review of antibiotic use in food animals: perspective, policy, and potential. Public Health Rep. 2012;127:4-22. 
5. Shade A, Peter H, Allison SD, Baho DL, Berga M, Bürgmann $H$, et al. Fundamentals of microbial community resistance and resilience. Front Microbiol. 2012:3:417.

6. Hahn M. The rising threat of fungicide resistance in plant pathogenic fungi: Botrytis as a case study. J Chem Biol. 2014;7:133-41.

7. Schaeffer RN, Vannette RL, Brittain C, Williams NM, Fukami T. Non-target effects of fungicides on nectar-inhabiting fungi of almond flowers. Environ. Microbiol. Rep. 2017;9:79-84.

8. Zubrod JP, Bundschuh M, Arts G, Brühl CA, Imfeld G, Knäbel A, et al. Fungicides: An Overlooked Pesticide Class? Environ Sci Technol. 2019;53:3347-65.

9. Delmas CEL, Dussert Y, Delière L, Couture C, Mazet ID, Richart Cervera S, et al. Soft selective sweeps in fungicide resistance evolution: recurrent mutations without fitness costs in grapevine downy mildew. Mol. Ecol. 2017;26:1936-51.

10. McDonald MC, Renkin M, Spackman M, Orchard B, Croll D, Solomon PS, et al. Rapid parallel evolution of azole fungicide resistance in Australian populations of the wheat pathogen Zymoseptoria tritici. Appl Environ Microbiol. 2019;85: e01908-e01918.

11. Riat A, Plojoux J, Gindro K, Schrenzel J, Sanglard D. Azole Resistance of environmental and clinical Aspergillus fumigatus isolates from Switzerland. Antimicrob Agents Chemother. 2018;62:e02088-e02017.

12. Verweij PE, Snelders E, Kema GHJ, Mellado E, Melchers WJG. Azole resistance in Aspergillus fumigatus: a side-effect of environmental fungicide use? Lancet Infect Dis. 2009;9:789-95.

13. Wise $K$, Mueller D Are fungicides no longer just for fungi? An analysis of folia fungicide use in corn. APSnet Features doi 2011; 10.

14. Kandel YR, Hunt C, Ames K, Arneson N, Bradley CA, Byamukama E, et al. MetaAnalysis of Soybean Yield Response to Foliar Fungicides Evaluated from 2005 to 2018 in the United States and Canada. Plant Dis. 2021;105:1382-9.

15. Imfeld $G$, Vuilleumier $S$. Measuring the effects of pesticides on bacterial communities in soil: A critical review. Eur J Soil Biol. 2012;49:22-30.

16. Fournier B, Dos Santos SP, Gustavsen JA, Imfeld G, Lamy F, Mitchell EAD, et al. Impact of a synthetic fungicide (fosetyl-Al and propamocarb-hydrochloride) and a biopesticide (Clonostachys rosea) on soil bacterial, fungal, and protist communities. Sci Total Environ. 2020;738:139635.

17. Morton V, Staub T A Short History of Fungicides. APSnet Feature Articles. 2008.

18. Brent KJ, Hollomon DW Fungicide resistance in crop pathogens: how can it be managed? 2007. FRAC Monogr. No. 1, Global Prot. Fed.

19. Perazzolli M, Antonielli L, Storari M, Puopolo G, Pancher M, Giovannini O, et al. Resilience of the natural phyllosphere microbiota of the grapevine to chemical and biological pesticides. Appl Environ Microbiol. 2014;80:3585-96.

20. Knorr K, Jørgensen LN, Nicolaisen M. Fungicides have complex effects on the wheat phyllosphere mycobiome. PLoS One. 2019;14:e0213176.

21. Sapkota R, Knorr K, Jørgensen LN, O'Hanlon KA, Nicolaisen M. Host genotype is an important determinant of the cereal phyllosphere mycobiome. New Phytol. 2015;207:1134-44.

22. Southwell RJ, Brown JF, Welsby SM. Microbial interactions on the phylloplane of wheat and barley after applications of mancozeb and triadimefon. Australasian Plant Pathol. 1999;28:139.

23. Dickinson $\mathrm{CH}$, Wallace $\mathrm{B}$. Effects of late applications of foliar fungicides on activity of micro-organisms on winter wheat flag leaves. Trans $\mathrm{Br}$ Mycological Soc. 1976;67:103-12.

24. Freimoser FM, Rueda-Mejia MP, Tilocca B, Migheli Q. Biocontrol yeasts: mechanisms and applications. World J Microbiol Biotechnol. 2019;35:154.

25. Fonseca Á, Inácio J Phylloplane Yeasts. In: Péter G, Rosa C (eds). Biodiversity and Ecophysiology of Yeasts. 2006. Springer Berlin Heidelberg, Berlin, Heidelberg, pp. 263-301.

26. Cobban A, Edgcomb VP, Burgaud G, Repeta D, Leadbetter ER. Revisiting the pinkred pigmented basidiomycete mirror yeast of the phyllosphere. Microbiologyopen. 2016;5:846-55.

27. Cadez N, Zupan J, Raspor P. The effect of fungicides on yeast communities associated with grape berries. FEMS Yeast Res. 2010;10:619-30.

28. Schaeffer RN, Mei YZ, Andicoechea J, Manson JS, Irwin RE. Consequences of a nectar yeast for pollinator preference and performance. Funct Ecol. 2017;31:613-21.

29. Agler MT, Ruhe J, Kroll S, Morhenn C, Kim S-T, Weigel D, et al. Microbial Hub Taxa Link Host and Abiotic Factors to Plant Microbiome Variation. PLoS Biol. 2016;14:e1002352.

30. Tilman D. The ecological consequences of changes in biodiversity: a search for general principles. Ecology. 1999;80:1455.

31. Ripple WJ, Beschta RL. Linking wolves and plants: aldo leopold on trophic cascades. BioScience. 2005;55:613.

32. Sahasrabudhe $S$, Motter AE. Rescuing ecosystems from extinction cascades through compensatory perturbations. Nat Commun. 2011;2:170.

33. Zhou J, Deng Y, Luo F, He Z, Tu Q, Zhi X. Functional molecular ecological networks. mBio. 2010;1:e00169-e00210.

34. Wagg C, Schlaeppi K, Banerjee S, Kuramae EE, van der Heijden MGA. Fungalbacterial diversity and microbiome complexity predict ecosystem functioning. Nat Commun. 2019;10:4841.
35. Claassen R, Bowman M, McFadden J, Smith D, Wallander S. Tillage intensity and conservation cropping in the United States. US Dep Agric Bull Econ Rese Ser. 2018;197:1-21.

36. Gdanetz K, Trail F. The wheat microbiome under four management strategies, and potential for endophytes in disease protection. Phytobiomes J. 2017;1:158-68.

37. Longley R, Noel ZA, Benucci GMN, Chilvers MI, Trail F, Bonito G. Crop management impacts the soybean microbiome. Front Microbiol. 2020;11:1116.

38. Sułowicz S, Cycoń M, Piotrowska-Seget Z. Non-target impact of fungicide tetraconazole on microbial communities in soils with different agricultural management. Ecotoxicology. 2016;25:1047-60.

39. Karlsson I, Friberg H, Steinberg C, Persson P. Fungicide effects on fungal community composition in the wheat phyllosphere. PLoS One. 2014;9:e111786.

40. Robertson GP, Hamilton SK Long-term ecological research at the Kellogg Biological Station LTER site. The ecology of agricultural landscapes: Long-term research on the path to sustainability 2015; 1-32.

41. Fehr WR, Caviness CE, Burmood DT, Pennington JS. Stage of development descriptions for soybeans, Glycine max (L.) Merrill 1. Crop Sci. 1971;11:929-31.

42. Gdanetz K, Noel Z, Trail F. Influence of plant host and organ, management strategy, and spore traits on microbiome composition. Phytobiomes J. 2021;5:202-19.

43. Lundberg DS, Yourstone $S$, Mieczkowski $P$, Jones CD, Dangl JL. Practical innovations for high-throughput amplicon sequencing. Nat Methods. 2013;10:999-1002.

44. Bowsher AW, Benucci GMN, Bonito G, Shade A. Seasonal Dynamics of Core Fungi in the Switchgrass Phyllosphere, and Co-Occurrence with Leaf Bacteria. Phytobiomes J. 2021;5:60-68.

45. Oksanen J, Blanchet FG, Friendly M, Kindt R, Legendre P, McGlinn D, et al. vegan: Community Ecology Package v. 2.5-7. 2020

46. Anderson MJ, Willis TJ. Canonical analysis of principle coordinates: a useful method of constrained ordination for ecology. Ecology. 2003;84:511-25.

47. Mandal S, Van Treuren W, White RA, Eggesbø M, Knight R, Peddada SD. Analysis of composition of microbiomes: a novel method for studying microbial composition. Microb Ecol Health Dis. 2015;26:27663.

48. Shade A, Stopnisek N. Abundance-occupancy distributions to prioritize plant core microbiome membership. Curr Opin Microbiol. 2019;49:50-58.

49. Liaw A, Wiener $M$. Classification and regression by randomForest. $R$ News. 2002;2:18-22.

50. Kursa MB, Rudnicki WR. Feature selection with the Boruta package. J Stat Softw. 2010;36:1-13.

51. Kurtz ZD, Müller CL, Miraldi ER, Littman DR, Blaser MJ, Bonneau RA. Sparse and compositionally robust inference of microbial ecological networks. PLoS Comput Biol. 2015;11:e1004226.

52. Lindow SE, Brandl MT. Microbiology of the phyllosphere. Appl Environ Microbiol. 2003;69:1875-83.

53. Wang K, Sipilä TP, Overmyer K. The isolation and characterization of resident yeasts from the phylloplane of Arabidopsis thaliana. Sci Rep. 2016;6:39403.

54. Sun PF, Fang WT, Shin LY, Wei JY, Fu SF, Chou JY. Indole-3-acetic acid-producing yeasts in the phyllosphere of the carnivorous plant Drosera indica L. PLoS One. 2014;9:e114196.

55. Yurkov AM, Kurtzman CP. Three new species of Tremellomycetes isolated from maize and northern wild rice. FEMS Yeast Res. 2019;19:foz004.

56. Sommermann L, Geistlinger J, Wibberg D, Deubel A, Zwanzig J, Babin D, et al. Fungal community profiles in agricultural soils of a long-term field trial under different tillage, fertilization and crop rotation conditions analyzed by highthroughput ITS-amplicon sequencing. PLOS ONE. 2018;13:e0195345.

57. Li A-H, Yuan F-X, Groenewald M, Bensch K, Yurkov AM, Li K, et al. Diversity and phylogeny of basidiomycetous yeasts from plant leaves and soil: proposal of two new orders, three new families, eight new genera and one hundred and seven new species. Stud Mycol. 2020;96:17-140.

58. Gilbert DG. Dispersal of yeasts and bacteria by Drosophila in a temperate forest. Oecologia. 1980;46:135-7.

59. Starmer WT, Peris F, Fontdevila A. The transmission of yeasts by Drosophila buzzatii during courtship and mating. Animal Behaviour. 1988;36:1691-5.

60. Murrell EG. Can agricultural practices that mitigate or improve crop resilience to climate change also manage crop pests? Curr Opin Insect Sci. 2017;23:81-88.

61. Latin R A Practical Guide to Turfgrass Fungicides. 2017.

62. Banerjee S, Walder F, Büchi L, Meyer M, Held AY, Gattinger A, et al. Agricultural intensification reduces microbial network complexity and the abundance of keystone taxa in roots. ISME J. 2019;13:1722-36.

63. Schmidt JE, Kent AD, Brisson VL, Gaudin ACM. Agricultural management and plant selection interactively affect rhizosphere microbial community structure and nitrogen cycling. Microbiome. 2019;7:146.

64. Brockhurst MA, Buckling A, Gardner A. Cooperation peaks at intermediate disturbance. Curr Biol. 2007;17:761-5.

65. Brockhurst MA, Habets MGJL, Libberton B, Buckling A, Gardner A. Ecological drivers of the evolution of public-goods cooperation in bacteria. Ecology. 2010;91:334-40. 
10

66. Kwak M-J, Jeong H, Madhaiyan M, Lee $Y$, Sa T-M, Oh TK, et al. Genome information of Methylobacterium oryzae, a plant-probiotic methylotroph in the phyllosphere. PLoS One. 2014;9:e106704.

67. Yoshida S, Hiradate S, Koitabashi M, Kamo T, Tsushima S. Phyllosphere Methylobacterium bacteria contain UVA-absorbing compounds. J Photochem Photobiol B. 2017;167:168-75.

68. Grady KL, Sorensen JW, Stopnisek N, Guittar J, Shade A. Assembly and seasonality of core phyllosphere microbiota on perennial biofuel crops. Nat Commun. 2019;10:4135.

69. Delmotte N, Knief C, Chaffron S, Innerebner G, Roschitzki B, Schlapbach R, et al. Community proteogenomics reveals insights into the physiology of phyllosphere bacteria. Proc Natl Acad Sci USA. 2009;106:16428-33.

\section{ACKNOWLEDGEMENTS}

This work was supported by the NIFA grant MICL08541 from the USDA National Institute of Food and Agriculture to GB, FT, and MC and USDA MICL02416 to GB. Support for this research was also provided by the NSF Long-term Ecological Research Program (DEB 1832042) at the Kellogg Biological Station and by Michigan State University AgBioResearch. This publication was made possible by a predoctoral training award to RL from Grant Number T32-GM110523 from National Institute of General Medical Sciences of the National Institutes of Health. We would also like to thank Elizabeth Gall for field and lab assistance. We would like to thank Alex Witte for help with applying the fungicides.

\section{AUTHOR CONTRIBUTIONS}

ZAN and RL contributed equally to this work. MIC, FT, and GB designed experiments. MIC applied fungicides. MIC, FT, GB, and RL collected samples. ZAN and RL prepared amplicon libraries and generated sequencing data. ZAN, RL, GMNB, and GB wrote and edited the manuscript, with contributions from MIC and FT. ZAN, RL, GMNB processed sequencing data and analyzed data. ZAN generated figures.

\section{COMPETING INTERESTS}

The authors declare no competing interests.

\section{ADDITIONAL INFORMATION}

Supplementary information The online version contains supplementary material available at https://doi.org/10.1038/s43705-022-00103-w.

Correspondence and requests for materials should be addressed to Gregory Bonito.

Reprints and permission information is available at http://www.nature.com/ reprints

Publisher's note Springer Nature remains neutral with regard to jurisdictional claims in published maps and institutional affiliations.

(i) Open Access This article is licensed under a Creative Commons Attribution 4.0 International License, which permits use, sharing, adaptation, distribution and reproduction in any medium or format, as long as you give appropriate credit to the original author(s) and the source, provide a link to the Creative Commons license, and indicate if changes were made. The images or other third party material in this article are included in the article's Creative Commons license, unless indicated otherwise in a credit line to the material. If material is not included in the article's Creative Commons license and your intended use is not permitted by statutory regulation or exceeds the permitted use, you will need to obtain permission directly from the copyright holder. To view a copy of this license, visit http://creativecommons. org/licenses/by/4.0/.

(c) The Author(s) 2022 